\title{
Water Spray Dynamics in Fire Flows
}

\author{
VASILY NOVOZHILOV \\ Fire Safety Engineering Research and Technology Centre \\ University of Ulster \\ 27B08 School of the Built Environment \\ Newtownabbey, BT37 0QB, United Kingdom
}

\begin{abstract}
Lagrangian model of droplet movement in axisymmetrical fluid flow is considered in application to water sprays in active fire control systems, such as sprinklers, aerosols and water mists. In contrast to previously known models equations of droplet motion are integrated in much more general form, with axial flow velocity being arbitrary function of two spatial coordinates. Second major advantage of the model is that it fully accounts for the droplet evaporation. Explicit formulas are derived for vaporization rate density and drag force at any location in the flow. Such formulas are directly applicable in a number of fire engineering problems, for example in estimating compartment cooling rates by water sprays, and in studying smoke layer destabilization by sprinkler or water mist sprays.
\end{abstract}

KEYWORDS: suppression, water sprays, lagrangian models

\section{NOMENCLATURE LISTING}

$\begin{array}{ll}C_{D} & \text { drag coefficient } \\ d & \text { droplet diameter } \\ f & \text { body force } \\ F_{d} & \text { drag force } \\ g & \text { gravity acceleration } \\ k_{e v} & \text { evaporation constant } \\ \dot{m} & \text { vaporization rate } \\ r & \text { radial coordinate } \\ R e & \text { Reynolds number } \\ t_{e v} & \text { evaporation time }\end{array}$

\section{special symbols}

$\sim \quad$ wave - dimensional variables; unwaved variables are non-dimensional

\section{INTRODUCTION}

Despite wide-spread attempts to apply CFD models to analysis of multiphase flows emerging in fire suppression, their true accuracy, and consequently blind prediction capabilities often cannot be estimated. The drive of the last decades [1] behind the development of multiphase models for fire engineering applications has largely leveled off and amounts to a large extent to application of Fire Dynamics Simulator, e.g. [2,3]. There are several exceptions, most notable being reported in the studies [4-8]. Nmira et al., [4] porpose Eulerian-Eulerian model for multiphase spray flows in fire applications. The flow treatment is based on the RANS approach. Collin et al., [6] uses well-established Eulerian-Lagrangian approach. Unfortunately, the details of gas flow treatment are not presented, but impression from both the studies $[4,6]$ suggests that they use somewhat obsolete methods of treating flow turbulence. The studies [5,7] present relevant, but not directly related to the topic of the present paper studies, namely on multiphase Radiative Transfer Equation [5] and on water film flows over solid surfaces [7]. In the light of what has been just said about the studies $[4,6]$ the best progress, in the author opinion, has been made by Luo and co-workers, e.g [8]. The major advantage of their approach is high accuracy of numerical treatment of turbulence in multiphase systems with the LES methodology. Such treatment is of paramount importance in the considered problem. On the other hand, their studies emphasize challenges which still persist. Even with the very accurate approach all the relevant scales cannot be resolved, and the absence of coherent subgrid-scale suppression model (based on solid combustion theory principles) does not allow confident prediction of local suppression events in the flame to be made. 
A major obstacle for proliferation of such sophisticated methods into industry is high cost associated with challenging model development and validation (manpower costs) and actual computations demanding supercomputer resources. Not surprisingly, to the best of our knowledge, models comparable in accuracy to [8] are not used in fire engineering practice. It is clear that further fundamental developments of CFD models for fire suppression engineering are very much hindered by aforementioned high costs and efforts required for a descent progress in subgrid-scale suppression model development.

More simple analytical solutions, essentially supplementing zone fire models in terms of action of suppression systems, are still in extensive use. These are very helpful when quick solutions are required for practical problems, such as predicting smoke layer cooling [9] or its destabilization by sprinkler sprays [10,11]. These models typically solve momentum equation for the vertical droplet velocity component with the drag force proportional to the square of that component. Severe limitations of such existing treatments are

(i) ignorance of carrying (background) flow velocity, i.e. surrounding media is assumed to be quiescent. This assumption may be reasonable within large-volume smoke layer, but is clearly violated when droplets are injected against fire plume flow, or into the ceiling jet. Consequently, the drag on droplets is not correctly estimated, as there is no correct estimation of the relative droplet/gas velocity.

(ii) drag coefficient is taken as constant, that is not varying with the Reynolds number. Such treatment excludes quickly decelerating droplets approaching small velocities. This regime, nevertheless, is extremely important, for example for small quickly evaporating droplets of water mists. In reality, any droplet approaches zero velocity as it approaches full evaporation.

(iii) evaporation of droplets is neglected. This assumption further aggravates limitations indicated under the points (i) and (ii).

It is desirable to develop spray dynamics models that, while preserving simplicity of solutions, approach capabilities of CFD technology. The present paper offers first step in this direction by significantly relaxing assumptions employed in [9-11] and a number of similar models. Specifically, we consider spray dynamics in the carrying fluid flow with axial velocity changing as an arbitrary function of two spatial variables. Drag coefficient varies realistically with Reynolds number in a full range of change of the latter parameter. Further, and importantly, droplet dynamics is fully coupled with the droplet evaporation process.

Model development and solutions for emerging equations are presented. We discuss further the two applications. One employs elements of qualitative analysis of the model and leads to discussion of an optimum diameter for fire fighting. As a second application, we present derivation of formulas that allow calculation (within the framework of the proposed model) of droplet vaporization rate density and drag exerted on gas/smoke at any location in the flow.

\section{MATHEMATICAL MODEL}

We start with the conventional Lagrangian equation of the droplet motion $[12,13]$.

$\frac{d \tilde{\vec{V}}}{d \tilde{t}}=-\frac{3}{4} \frac{\tilde{\rho}}{\tilde{\rho}_{p}} \frac{C_{D}}{\tilde{d}}(\tilde{\vec{V}}-\tilde{\vec{U}})|\tilde{\vec{V}}-\tilde{\vec{U}}|+\tilde{\vec{f}}$

where $\tilde{\bar{V}}=\tilde{\bar{V}}(\tilde{t}) \quad \tilde{\vec{U}}=\tilde{\vec{U}}(\tilde{\vec{x}}, \tilde{t}) \quad \tilde{\vec{f}}=\tilde{\vec{f}}(\tilde{\vec{x}}, \tilde{t})$

Drag coefficient $C_{D}$ is a well-studied (for spherical particles) function of the Reynolds number $\operatorname{Re}_{p}=\tilde{d} \cdot|\tilde{\vec{V}}-\tilde{\vec{U}}| \cdot \tilde{v}^{-1}$ which can be described, for example, by the following mostly used dependence [13]

$C_{D}=\frac{24}{\operatorname{Re}_{p}}\left(1+\frac{\operatorname{Re}_{p}^{2 / 3}}{6}\right), \quad \operatorname{Re}_{p}<10^{3}$ 
In the present study, the experimental dependence (2) is approximated by the following analytical function

$$
C_{D}=50 / \operatorname{Re}_{p}, \quad \operatorname{Re}_{p}<10^{3}
$$

It is easy to check that the range of particle Reynolds numbers in $(2,3)$ covers the injection conditions for typical ranges of droplet sizes in sprinkler sprays and mists. Accuracy of this approximation may be evaluated from Fig.1. While the relative deviation from the correlation (2) may reach $50 \%$ in certain regions, it should be noted that the same level of discrepancy is observed between the two alternative experimental correlations presented in [13]. The latter fact suggests that the accuracy of the drag coefficient approximation adopted in the present study is comparable to the overall accuracy of analytical correlations adopted at present time for describing experimental measurements of drag coefficients. Further, implication of accuracy of the approximation (3) will become clear below when validation of obtained results is presented.

Upon approximation (3), the equation (1) takes the form

$\frac{d \widetilde{\vec{V}}}{d \widetilde{t}}=-\widetilde{\alpha} \frac{1}{\widetilde{d}^{2}}(\widetilde{\vec{V}}-\widetilde{\vec{U}})+\widetilde{\vec{f}}$

where $\tilde{\alpha}=75 / 2 \tilde{v} \tilde{\rho} \tilde{\rho}_{p}^{-1}$.

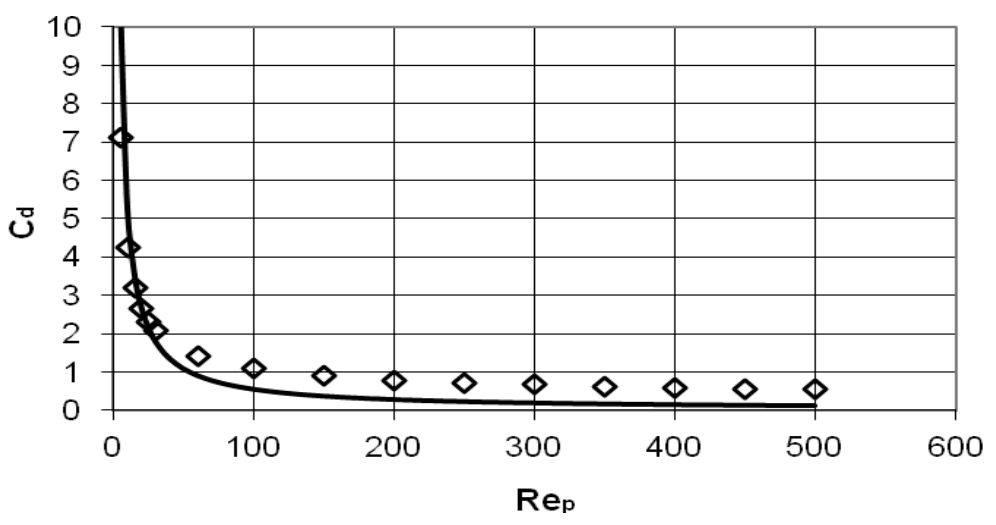

Fig. 1. Approximation of the drag coefficient dependence on the particle Reynolds number. Solid line - approximate dependence (3); $\diamond$ - experimental correlation (2).

Some further assumptions are required in order to identify integrable cases of (4). We shall assume that the flow velocity field is known and unaffected by particle motion. Therefore, the treatment is restricted to oneway coupling between the phases, i.e. strictly applicable to "weak" particle concentrations and dilute sprays. This assumption will be gradually relaxed in further studies. It is assumed in the present paper that the background flow is cylindrically symmetrical and parallel to the axis of symmetry (Fig. 2). This assumption is reasonable in many fire applications, for example in a core region of a fire plume. Importantly, it should be noted that the model will be equally applicable as long as the carrying flow is parallel and depends on the two spatial coordinates only. Such will be a situation during spray injection into the boundary layer, against a flame spreading on a flat surface, or into a ceiling jet.

We assume that external body force is invariant in space and time.

The assumptions results in the following equations for the two unknown components of the particle velocity

$\frac{d \tilde{V}_{r}}{d \tilde{t}}=-\tilde{\alpha} \frac{1}{\tilde{d}^{2}}\left(\tilde{V}_{r}-\tilde{U}_{r}\right)+\tilde{f}_{r} \quad \frac{d \tilde{V}_{x}}{d \tilde{t}}=-\tilde{\alpha} \frac{1}{\tilde{d}^{2}}\left(\tilde{V}_{x}-\tilde{U}_{x}\right)+\tilde{f}_{x}$

where $\tilde{V}_{r}=\tilde{V}_{r}(\tilde{t}) \quad \tilde{V}_{x}=\tilde{V}_{x}(\tilde{t}) \quad \tilde{U}_{r} \equiv 0 \quad \tilde{U}_{x} \equiv \tilde{U}_{x}(\tilde{r}, \tilde{x}) \quad \tilde{f}_{r}=\tilde{f}_{r}(\tilde{m}) \quad \tilde{f}_{r}=\tilde{f}_{r}(\tilde{m})$ 
The present model is primarily intended for application in the hot ("core") regions of fire plumes. Evaporation of droplets is assumed to occur in a hot environment, such that the " $d^{2}-$ " law $\tilde{d}_{0}^{2}-\tilde{d}^{2}(\tilde{t})=\tilde{k}_{e v} \cdot \tilde{t}$ [12] is applicable. This relation describes steady-state evaporation conditions. Droplet vanishes (evaporates completely) at the time $\tilde{t}_{e v}=\tilde{d}_{0}^{2} / \tilde{k}_{e v}$. This circumvents the necessity to consider droplet energy equation in detail. Straightforward estimation shows that at $200^{\circ} \mathrm{C}$, for a range of droplet sizes relevant to fire sprinklers, droplet heat-up time is only about $10 \%$ of the droplet lifetime under steady-state conditions. The time ratio between the two phases (i.e. between the heat-up and steady-state evaporation times) decreases quickly as background temperature rises.

In general, droplet heat-up phase can be taken into account in the model, and this will be done in subsequent publications.

The equations (5) are transformed further into non-dimensional form. For this purpose, $\tilde{t}_{e v}$ is used as a time scale, and the non-dimensional time is defined as $\tau=1-\frac{\tilde{t}}{\tilde{t}_{e v}}$. Therefore, it is being counted backwards from the initial position ( $\tau=1)$ to complete evaporation $(\tau=0)$. It is easy to see that the non-dimensional time is simply $\tau=\frac{\tilde{d}^{2}}{\tilde{d}_{0}^{2}}$, i.e. is equal to the ratio between the current and the initial droplet diameters.

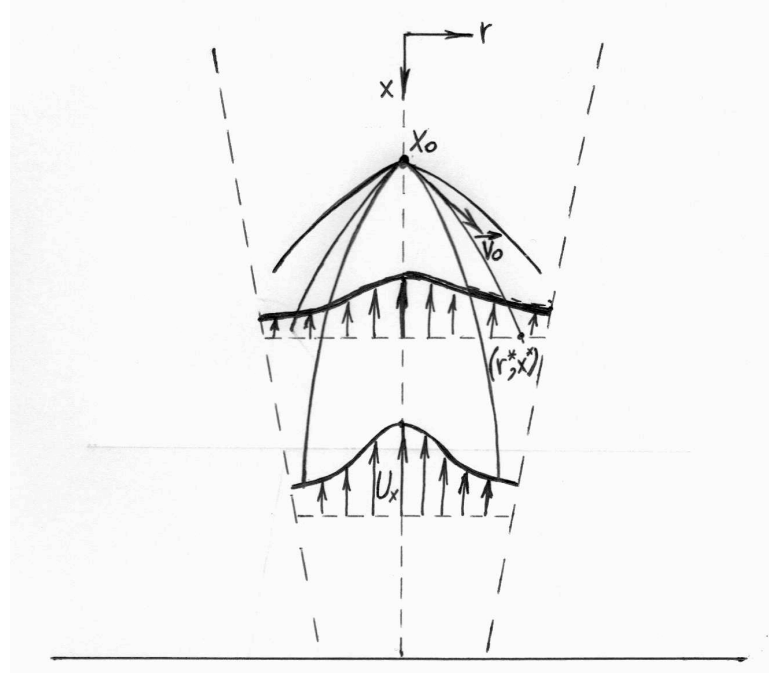

Fig. 2. Axisymmetrical flow with spray injection.

For flexibility, the two spatial scales are used, $\tilde{R}$ in the radial direction and $\tilde{L}$ longitudinal direction. This may be convenient in problems with high aspect ratios. In the other cases both scales may be taken to be equal $\tilde{R}=\tilde{L}$.

The velocity $\overrightarrow{\tilde{V}}_{0}\left(\tilde{V}_{r}^{0}, \tilde{V}_{x}^{0}\right)$ is the droplet injection (initial) velocity. Scales for velocity components are their initial (injection) values $\tilde{V}_{r}^{0}, \tilde{V}_{x}^{0}$; they are assumed to be different from zero, which is the case in nearly all practical situations. (In case one or both initial velocity components need be set to zero, characteristic flow velocity may be used instead). Vector $\left(\tilde{f}_{r}, \tilde{f}_{x}\right)$ is generally an arbitrary body force vector; in applications below $\tilde{f}_{r} \equiv 0$, $\tilde{f}_{x}=\tilde{g}$, i.e. this describes gravity acceleration.

The equations (5) are written in the following non-dimensional form 
$\frac{d V_{r}}{d \tau}=\frac{\beta}{\tau} V_{r}-f_{r}^{2}, \frac{d V_{x}}{d \tau}=\frac{\beta}{\tau} V_{x}-\left(\frac{\beta}{\tau} U_{x}+f_{x}^{2}\right)$

where $\beta=\frac{\tilde{\alpha}}{\tilde{k}_{e v}} \quad f_{r}^{2}=\frac{\tilde{d}_{0}^{2}}{\tilde{k}_{e v} \tilde{V}_{r}^{0}} \tilde{f}_{r} \quad f_{x}^{2}=\frac{\tilde{d}_{0}^{2}}{\tilde{k}_{e v} \tilde{V}_{x}^{0}} \tilde{f}_{x}$.

According to adopted scaling, the initial conditions are $V_{r}(1)=V_{x}(1)=1$.

\section{RESULTS AND DISCUSSION}

Solution of the first (radial component) of equations (6) is straightforward

$V_{r}(\tau)=\left[1+\frac{1}{(1-\beta)} f_{r}^{2}\left(1-\tau^{(1-\beta)}\right)\right] \tau^{\beta}$

This solution provides radial position of the droplet at any time

$r(\tau)=r_{0}+p_{1} \int_{\tau}^{1} V_{r}(\xi) d \xi \quad p_{1}=\frac{\tilde{V}_{r}^{0}}{\tilde{R}} \frac{\tilde{d}_{0}^{2}}{\tilde{k}_{e v}}$

It is reminded that the non-dimensional time is counted backwards, from 1 to 0 along the droplet trajectory. Hence, the current position is obtained by velocity integration over $[\tau, 1]$; expression for the constant $p_{1}$ is obtained upon relevant non-dimensionalisation of the dimensional counterpart of (8).

resulting in

$r(\tau)=p_{1}\left[\frac{1}{(1+\beta)}\left(1+\frac{f_{r}^{2}}{(1-\beta)}\right)\left(1-\tau^{(1+\beta)}\right)-\frac{1}{2} \frac{f_{r}^{2}}{(1-\beta)}\left(1-\tau^{2}\right)\right]$

This describes partially the time dependence of the $U_{x}$ - term of the second (axial component) of equations (6), i.e.

$U_{x}=U_{x}(r(\tau), x(\tau))$

where $r(\tau)$ is given by (9), but the dependence $x(\tau)$ is determined, unfortunately, by the $V_{x}$ - solution itself:

$x(\tau)=x_{0}+p_{2} \int_{\tau}^{1} V_{x}(\xi) d \xi \quad p_{2}=\frac{\tilde{V}_{x}^{0}}{\tilde{L}} \frac{\tilde{d}_{0}^{2}}{\tilde{k_{e v}}}$

The latter circumstance ensues non-linearity of the $V_{x}$ - equation and thus presents major challenge for its analytical solution. Equation (11) has exactly the same position-velocity relation meaning as (8) above.

Before discussing solution of the equation (6.2) in detail, we consider an application of the proposed model rendering itself to relatively simple analysis.

\section{Optimum droplet diameter for fire fighting}

Design of sprays for efficient fire suppression is a matter of paramount importance. It is well realized that droplet sizes of the spray must be in some sense "optimal" in order to achieve desired performance. Notion of "optimum droplet size" has been discussed in various ways in the literature [14-16]. Despite the fact that 
optimality may be understood and defined in different ways, we demonstrate in this section how the proposed model may be applied to find optimum spray diameter, using one of possible definitions.

Specifically, for the sprays that are designed to suppress predominantly gaseous diffusion flames (e.g. water mists), the following criterion for optimality may be adopted: initial droplet diameter is optimum (under fixed injection velocity) if the droplet trajectory ends at the surface of the material due to complete evaporation. The meaning of this requirement is that it ensures droplet delivery into the flame zone and complete evaporation in this zone. Further, most of evaporation process may be expected to occur in the flaming zone since the droplet decelerates before vanishing at the surface. Therefore, the droplet should have large residence time in the flame, and consequently heat transfer and water vapor generation rates will be maximized.

Optimum droplet diameter is essentially an indicative integral parameter, which may serve as a guidance for mean droplet diameters of the spray required in particular suppression application. As such, its estimations are naturally obtained under certain simplified assumptions. It is assumed in the present study that the droplet is injected from the initial position $x=x_{0}$ downwards that is $\overrightarrow{\tilde{V}}_{0}=\left(0, \tilde{V}_{x}^{0}\right)$. It is also possible to obtain similar estimations for an any different initial velocity, however, this would require slightly modified arguments.

In any case, full analysis of droplet interaction with fire plume requires consideration of different droplet sizes and different initial velocities. Solution of equations (6) in general form is discussed in the next section, while the present section is restricted to simplified analysis with the downward velocity $\vec{V}_{0}=\left(0, \tilde{V}_{x}^{0}\right), \vec{V}_{0}=(0,1)$.

Analysis of the model under imposed criterion and initial velocity is possible upon invoking some qualitative considerations. Specifically, we note that the point of complete evaporation is also necessarily a point of zero velocity at the droplet trajectory. With this in mind, the exact solution of the equation (6.2) is not required, but rather locations of critical points of the droplet velocity field need be established.

Replacing time derivative with spatial derivative (using the kinematic identity $\frac{d \tilde{V}_{x}}{d \tilde{t}}=\frac{d \tilde{V}_{x}}{d \tilde{z}} \cdot \frac{d \tilde{z}}{d \tilde{t}}$ and its further non-dimensionalisation), the equation (6.2) is transformed to

$$
\tau V_{x} \frac{d V_{x}}{d x}=\frac{\beta}{\gamma}\left(U_{x}-V_{x}\right)+\frac{\tau}{\gamma} f_{x}^{2}
$$

where $\gamma=\frac{\tilde{t}_{e v}}{\tilde{L}} \tilde{V}_{x}^{0}=\frac{\tilde{d}_{0}^{2}}{\tilde{k}_{e v}} \frac{\tilde{V}_{x}^{0}}{\tilde{L}}$.

Integration of both sides of (12) over distance from the injection droplet position down to the surface results in

$$
\frac{1}{2}\left[\left.\tau V_{x}^{2}\right|_{0} ^{x_{0}}-\int_{0}^{x_{0}} V_{x}^{2} d \tau\right]=\frac{\beta}{\gamma} \int_{0}^{x_{0}}\left(U_{x}-V_{x}\right) d x+\frac{f_{x}^{2}}{\gamma} \int_{0}^{x_{0}} \tau d x
$$

where integration by parts is performed on the LHS.

The integral on the LHS can be transformed taking note of $d \tau=-\frac{1}{\gamma} \frac{1}{V_{x}} d x$, which follows from the " $d^{2}$-" law, to get

$$
\frac{1}{2}\left[1+\frac{1}{\gamma} \int_{0}^{x_{0}} V_{x} d \tau\right]=\frac{\beta}{\gamma} \int_{0}^{x_{0}} U_{x} d x-\frac{\beta}{\gamma} \int_{0}^{x_{0}} V_{x} d x+\frac{f_{x}^{2}}{\gamma} \int_{0}^{x_{0}} \tau d x
$$


Estimation of the integrals $\int_{0}^{x_{0}} V_{x} d \tau$ and $\int_{0}^{x_{0}} \tau d x$ is based on the following considerations. From the calculus mean value theorem $\int_{0}^{x_{0}} V_{x} d \tau \approx V_{x}\left(x_{*}^{1}\right) \cdot x_{0} ; \int_{0}^{x_{0}} \tau d x \approx \tau\left(x_{*}^{2}\right) \cdot x_{0}$ where $x_{*}^{1}, x_{*}^{2} \in[0,1]$. We approximate further $V_{x}\left(x_{*}^{1}\right)$ and $\tau\left(x_{*}^{2}\right)$ with (using median $\int_{0}^{x_{0}} V_{x} d \tau \approx \int_{0}^{x_{0}} \tau d x \approx \frac{x_{0}}{2}$ and therefore

$$
\frac{1}{2}\left[1+\frac{x_{0}}{2 \gamma}\right]=\frac{1}{\gamma}\left[\beta \int_{0}^{x_{0}} U_{x} d x+\frac{x_{0}}{2}\left(f_{x}^{2}-\beta\right)\right]
$$

Equation (15), by its derivation, holds for the droplet of the particular (optimum) initial diameter whose evaporation time is identical to the time taken by that droplet to reach the surface. This initial diameter is contained in the parameter $\gamma$. Solving, therefore, equation (15) for $\gamma$, and defining $\delta=\frac{\tilde{k}_{e v}}{\tilde{L} \tilde{V}_{x}^{0}}$ we obtain

$$
d_{0}^{o p t}=\sqrt{\delta\left[2 \beta \int_{0}^{x_{0}} U_{x} d x+\left(f_{x}^{2}-\left(\beta+\frac{1}{2}\right)\right) x_{0}\right]}
$$

The plume velocity profile $U_{x}(x)$ in (16) can be taken from the conventional experimental correlations in the plume and flame zones [17] and easily integrated analytically.

The result obtained using formula (16) is illustrated in Fig. 3 for a range of fire HRRs. Markers illustrate results obtained using accurate numerical integration of equation (6.2), as opposed to using formula (16) (solid line). The agreement is particularly good for smaller fires (up to about $1 \mathrm{MW}$ ), with the optimum diameter being slightly over-predicted for larger fires. Deviation between the results is due to all the assumptions made while arriving at (16). Major contribution to this discrepancy is believed to be mean value theorem approximations for the relevant integral terms. Overall, the deviation of results (in terms of relative error) predicted by formula (16) from "exact" numerical solution does not exceed $13 \%$ in the considered range of fire sizes

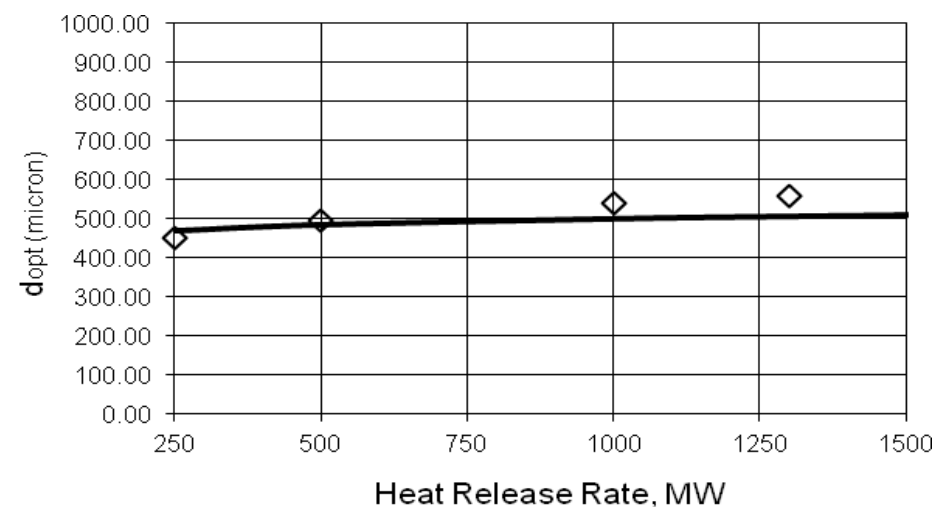

Fig. 3. Comparison between optimum droplet diameters predicted by analytical formula (16) and by numerical analysis. Solid line - formula (16); markers - numerical analysis. 


\section{General solution of equations (6)}

Equation (6.1) has been solved already, with the solution given by (7-9). Taking current position of the droplet

$x(\tau)=x_{o}+p_{2} \int_{\tau}^{1} V_{x}(s) d s$

as a new variable, equation (6.2) is written in the form

$\frac{d^{2} x}{d \tau^{2}}=\frac{\beta}{\tau} \frac{d x}{d \tau}+g(\tau)$

where

$g(\tau)=p_{2}\left(\frac{\beta}{\tau} U_{x}(r(\tau), x(\tau))+f_{x}^{2}\right)$

and the dependence $r(\tau)$ is given by (9).

Non-linear equation (18) can be solved iteratively, i.e. by solving progressively the series of equations

$\frac{d^{2} x_{n+1}}{d \tau^{2}}=\frac{\beta}{\tau} \frac{d x_{n+1}}{d \tau}+g_{n}(\tau) \quad n=0,1,2, \ldots$

where

$g_{n}(\tau)=p_{2}\left(\frac{\beta}{\tau} U_{x}\left(r(\tau), x_{n}(\tau)\right)+f_{x}^{2}\right), \quad n \geq 1 \quad g_{0}(\tau) \equiv 0$

The solution of the equation (20) can be written in the form

$x_{n+1}(\tau)=x_{0}+\int_{\tau}^{1}\left[\int_{s}^{1} \frac{g_{n}(\xi)}{\xi^{\beta}} d \xi+p_{2}\right] s^{\beta} d s$

Note that this solution satisfies the two initial conditions $x(1)=x_{0}$ and $\frac{d x}{d \tau}(1)=-p_{2}$. The form of the latter condition is a result of the choice of coordinate frame, Fig. 2, backward time change along the trajectory ( $x$ increases as $\tau$ decreases from 1 ), and the scaling $V_{x}(1)=1$.

The solution of (18) is obtained as the limit

$x(\tau)=x_{0}+\lim _{n \rightarrow \infty} \int_{\tau}^{1}\left[\int_{s}^{1} \frac{g_{n}(\xi)}{\xi^{\beta}} d \xi+p_{2}\right] s^{\beta} d s$

The model is validated by comparing in Fig. 4 solutions $(9,23)$ in the ideal plume convective flow generated by point source located $1 \mathrm{~m}$ below the point of droplet injection, with the trajectories obtained by direct numerical integration of equations $(1,2)$.. Such flow configuration corresponds essentially to Zukoski-type correlations for real fire plumes [17]. 


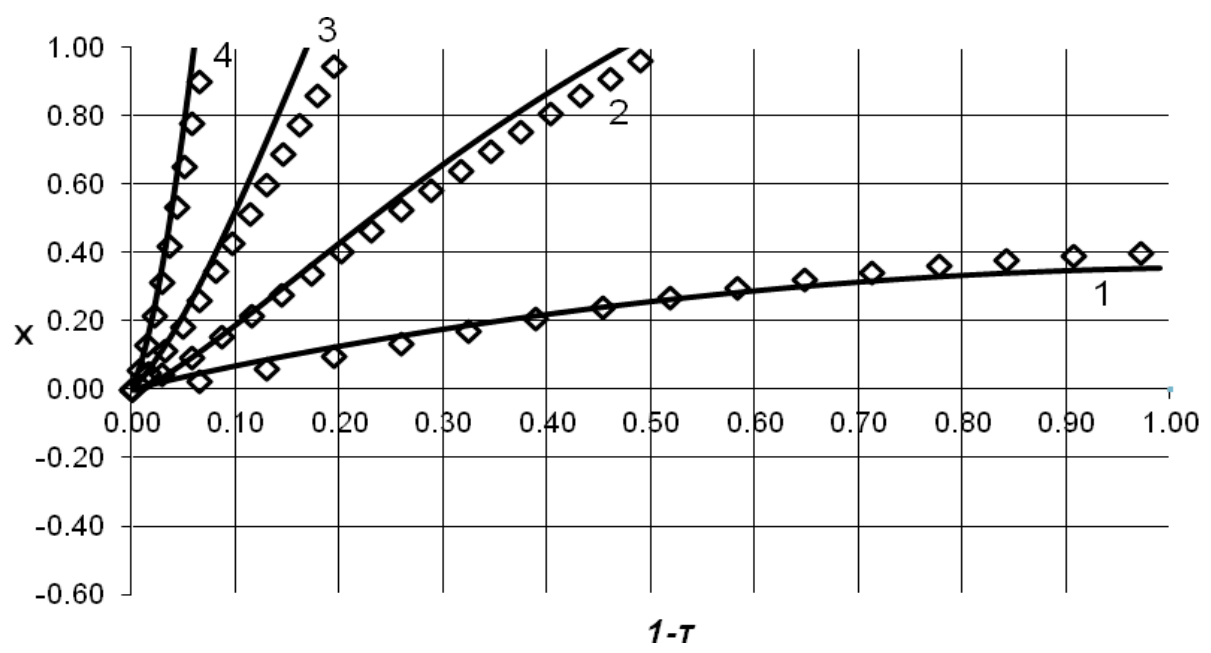

Fig. 4. Axial positions of injected droplets versus non-dimensional time $1-\tau$ along the trajectory. Solid lines analytical solution (present study); markers - numerical integration.

$1-\tilde{d}_{0}=200 \mu \mathrm{m} ; 2-\tilde{d}_{0}=300 \mu \mathrm{m} ; 3-\tilde{d}_{0}=400 \mu \mathrm{m} ; 4-\tilde{d}_{0}=600 \mu \mathrm{m}$

Generally, results in Fig. 4 demonstrate good accuracy of proposed analytical solutions (at least, in the range of parameters studied). Accuracy of the model can be improved further by considering piecewise approximation for the drag coefficient correlation (2).

\section{Integral properties of the spray}

Major integral properties of the spray that are of interest in fire engineering applications are distributions of vaporization rate and drag force on surrounding smoke/air.

Consider first of all droplets with trajectories reaching a particular point $\left(r^{*}, x^{*}\right)$ at time $0<\tau^{*}<1$, Fig. 2 .

Such droplets must have particular initial injection angles and droplet diameters $\left(\alpha, d_{0}\right)$.

From the formulas $(8,9,17,23)$

$$
\begin{aligned}
& p_{1}=\left(\tilde{R} \tilde{k}_{e v}\right)^{-1} \tilde{V}_{r}^{0} \tilde{d}_{0}^{2}=\left(\tilde{R} \tilde{k}_{e v}\right)^{-1}\left|V_{0}\right| \sin \alpha \cdot \tilde{d}_{0}^{2} \\
& p_{2}=\left(\tilde{L} \tilde{k}_{e v}\right)^{-1} \tilde{V}_{x}^{0} \tilde{d}_{0}^{2}=\left(\tilde{L} \tilde{k}_{e v}\right)^{-1}\left|V_{0}\right| \cos \alpha \cdot \tilde{d}_{0}^{2} \\
& d_{0}^{2}=\frac{r^{*} \tilde{R} \tilde{k}_{e v}}{V_{0} f_{1}\left(\tau^{*}\right)} \frac{1}{\sin \alpha}
\end{aligned}
$$

and therefore

$$
\operatorname{ctg}(\alpha)=\frac{\tilde{L}}{\tilde{R}} \frac{f_{1}\left(\tau^{*}\right)}{f_{2}\left(\tau^{*}\right)}\left(x^{*}-x_{0}\right)
$$


where the functions $f_{1}(\tau), f_{2}(\tau)$ are determined by (9,23). The equation (27) allows the injection angle $\alpha$, and the initial droplet diameter $d_{0}$ (from equation (26)) to be determined based on location $\left(r^{*}, x^{*}\right)$ and time $\tau^{*}$ as an input.

Calculation of the spray vaporization rate. First of all we note that non-dimensional (relative to the initial droplet mass $\tilde{m}_{0}=\frac{\pi}{6} \tilde{\rho}_{p} \tilde{d}_{0}^{3}$ ) vaporization rate of a single droplet along its trajectory may be written as

$\frac{d m_{p}}{d \tau}=\frac{d\left(\frac{\tilde{m}_{p}}{\tilde{m}_{0}}\right)}{d \tau}=\frac{d\left(\tau^{3 / 2}\right)}{d \tau}=\frac{3}{2} \sqrt{\tau}$

and becomes independent of $\tilde{d}_{0}$.

Dimensional vaporization rate is related to the latter as

$$
\frac{d \tilde{m}_{p}}{d \tilde{t}}=-\frac{\tilde{m}_{0}}{\tilde{t}_{e v}} \frac{d m_{p}}{d \tau}=-\frac{\pi}{4} \tilde{\rho}_{p} \tilde{k}_{e v} \tilde{d}_{0} \sqrt{1-\frac{\tilde{k}_{e v}}{\tilde{d}_{0}^{2}} \tilde{t}}
$$

Consider now non-dimensional droplet number rate $N\left(d_{0}\right)=\left(\tilde{d}_{0} \tilde{t}_{e v}\right) \tilde{N}\left(\tilde{d}_{0}\right)$ where $\tilde{N}\left(\tilde{d}_{0}\right)$ is conventional number rate density of droplets, i.e. density of number rate distribution over initial diameters.

Density of vaporization rate at a given location $\left(r^{*}, x^{*}\right)$ (Fig. 2,5), can be calculated by adding up contributions from all the particles travelling (at any time $0<\tau^{*}<1$ ) through small region surrounding this location, dividing by the volume of the region and taking the limit of the volume approaching zero. Droplets that enter the considered region are those whose initial conditions $\left(\alpha_{0}, d_{0}\right)$ belong to the vicinity of the root of equations $(26,27)$ at fixed $\left(r^{*}, x^{*}, \tau^{*}\right)$. Small region surrounding the root $\left(\alpha_{0}, d_{0}\right)$ maps onto the region surrounding location $\left(r^{*}, x^{*}\right)$ via the transformation $E \mapsto V_{\tau^{*}}(E): r^{*}=r^{*}\left(\alpha_{0}, d_{0}, \tau^{*}\right), x^{*}=x^{*}\left(\alpha_{0}, d_{0}, \tau^{*}\right)$ determined by the droplet trajectory. Jacobian of this transformation determines corresponding ratio of volumes (Fig. 5)

$$
\frac{V_{\tau}(E)}{E}=|J|=\left|\left(\begin{array}{cc}
\frac{\partial r^{*}}{\partial \alpha_{0}} & \frac{\partial r^{*}}{\partial d_{0}} \\
\frac{\partial x^{*}}{\partial \alpha_{0}} & \frac{\partial r^{*}}{\partial d_{0}}
\end{array}\right)\right|
$$

Non-dimensional density of local vaporization rate is calculated now, assuming angle invariance of the droplet size distribution, as

$$
\begin{aligned}
& \dot{m}_{d_{0}}\left(r^{*}, x^{*}\right)=\lim _{\varepsilon \rightarrow 0} \int_{0}^{1} \frac{\int_{\alpha_{0}-\varepsilon}^{\alpha_{0}+\varepsilon}\left(\int_{d_{0}-\varepsilon}^{d_{0}+\varepsilon}\left[N(d) \frac{d m_{p}}{d \tau}\right] d(d)\right) d \alpha}{V_{\tau^{*}}(E)} d \tau^{*}=3 \varepsilon \lim _{\varepsilon \rightarrow 0} \int_{0}^{1} \frac{\left(\int_{d_{0}-\varepsilon}^{d_{0}+\varepsilon}\left[N(d) \sqrt{\tau^{*}}\right] d(d)\right)}{V_{\tau^{*}}(E)} d \tau^{*}= \\
& =\lim _{\varepsilon \rightarrow 0}\left\{\frac{\varepsilon^{2}}{\varepsilon^{2} \mid J\left(d_{0}\right)} N\left(d^{*} \in\left(d_{0}-\varepsilon, d_{0}+\varepsilon\right)\right)\right\}=\left|J\left(d_{0}\right)\right|^{-1} N\left(d_{0}\right)
\end{aligned}
$$




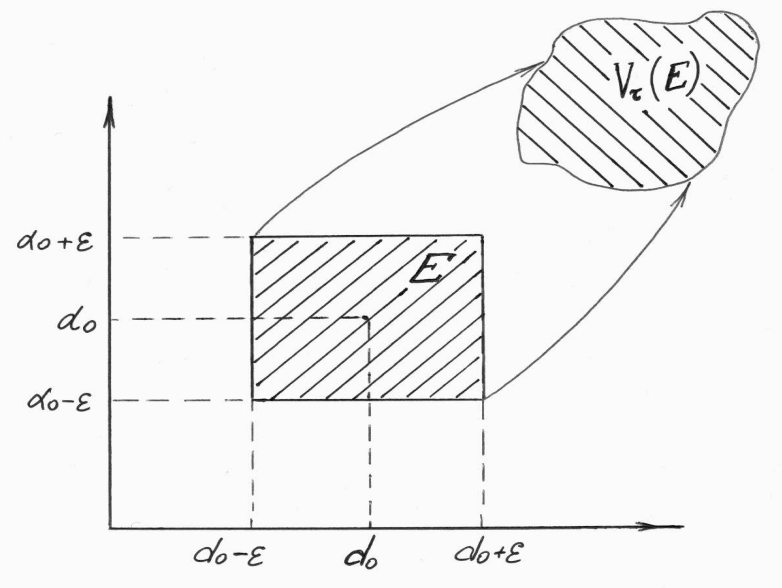

Fig. 5. Phase volume transformation between the "angle-diameter" space and the physical space. Formula (31) delivers contribution of droplets of a particular size; contribution of the whole spray is

$$
\dot{m}\left(r^{*}, x^{*}\right)=\int_{0}^{\infty}\left|J\left(d_{0}\right)\right|^{-1} N\left(d_{0}\right) d\left(d_{0}\right)
$$

Corresponding dimensional quantity may be restored invoking conventionally used density of particle size distribution in terms of volumetric fraction $\tilde{f}\left(\tilde{d}_{0}\right)$, its non-dimensional analogue $f\left(d_{0}\right)=\tilde{d}_{0} \tilde{f}\left(\tilde{d}_{0}\right)$, and total spray volume per unit time $V_{s}$. From the definitions of $N\left(d_{0}\right), f\left(d_{0}\right)$ and their dimensional counterparts it follows that

$$
f\left(d_{0}\right)=\frac{\pi}{6} \frac{\tilde{k}_{e v} \tilde{d}_{0}}{\tilde{V}_{s}} N\left(d_{0}\right)
$$

Therefore, the formula (32) gives

$\dot{m}\left(r^{*}, x^{*}\right)=\frac{6}{\pi} \frac{\tilde{V}_{s}}{\tilde{k}_{e v}} \int_{0}^{\infty} \frac{\left|J\left(d_{0}\right)\right|^{-1}}{\tilde{d}_{0}} f\left(d_{0}\right) d\left(d_{0}\right)$

or, in terms of dimensional $\tilde{f}\left(\tilde{d}_{0}\right)$,

$$
\dot{m}\left(r^{*}, x^{*}\right)=\frac{6}{\pi} \frac{\tilde{V}_{s}}{\tilde{k}_{e v}} \int_{0}^{\infty}\left|J\left(d_{0}\right)\right|^{-1} \tilde{f}\left(d_{0}\right) d\left(d_{0}\right)
$$

Invoking further the size-specific relation between non-dimensional and dimensional evaporation rates (29), we obtain finally

$$
\tilde{\dot{m}}\left(r^{*}, x^{*}\right)=\tilde{V}_{s} \int_{0}^{\infty}\left|J\left(\tilde{d}_{0}\right)\right|^{-1} \tilde{d}_{0} \tilde{f}\left(\tilde{d}_{0}\right) d\left(\tilde{d}_{0}\right)
$$


Calculation of the spray drag force. Drag exerted by the spray on surrounding smoke/air may be evaluated using similar methodology.

Following the original equation (4), drag force exerted on the gas at a particular location by the particle of the diameter $\tilde{d}_{0}$ is

$$
\tilde{\vec{F}}_{d_{0}}=\frac{27}{8} \pi \tilde{\rho}_{g} \tilde{v} \tilde{d}_{0}(\tilde{\vec{V}}-\tilde{\vec{U}})
$$

and corresponding non-dimensional force components may be defined as

$$
\vec{F}_{d_{0}}^{x}=\frac{1}{\tilde{\rho}_{g} \tilde{v} \tilde{d}_{0}\left|V_{x}^{0}\right|} \vec{F}_{d_{0}} \quad \vec{F}_{d_{0}}^{r}=\frac{1}{\tilde{\rho}_{g} \tilde{v} \tilde{d}_{0}\left|V_{r}^{0}\right|} \vec{F}_{d_{0}}
$$

It follows then from the results (7-23) that

$$
\vec{F}_{d_{0}}^{x}=\frac{27}{8} \pi\left(V_{x}-U_{x}\right) \quad \vec{F}_{d_{0}}^{r}=\frac{27}{8} \pi V_{r}
$$

with

$$
V_{x}=\frac{1}{p_{2}} \lim _{n \rightarrow \infty}\left[\int_{\tau}^{1} \frac{g_{n}(\xi)}{\xi^{\beta}} d \xi+p_{2}\right] \tau^{\beta}
$$

and $V_{r}$ given by (7).

Development analogous to (30-31) shows

$$
\begin{aligned}
& \vec{F}_{d_{0}}^{x}=\frac{3}{2} I_{1} \lim _{\varepsilon \rightarrow 0}\left\{\frac{\varepsilon^{2}}{\varepsilon^{2}\left|J\left(d_{0}\right)\right|} N\left(d^{*} \in\left(d_{0}-\varepsilon, d_{0}+\varepsilon\right)\right)\right\}=\frac{3}{2} I_{1}\left|J\left(d_{0}\right)\right|^{-1} N\left(d_{0}\right) \\
& \vec{F}_{d_{0}}^{r}=\frac{3}{2} I_{2} \lim _{\varepsilon \rightarrow 0}\left\{\frac{\varepsilon^{2}}{\varepsilon^{2}\left|J\left(d_{0}\right)\right|} N\left(d^{*} \in\left(d_{0}-\varepsilon, d_{0}+\varepsilon\right)\right)\right\}=\frac{3}{2} I_{2}\left|J\left(d_{0}\right)\right|^{-1} N\left(d_{0}\right) \\
& \vec{F}_{d}^{x}\left(r^{*}, x^{*}\right)=\frac{3}{2} I_{1} \int_{0}^{\infty}\left|J\left(d_{0}\right)\right|^{-1} N\left(d_{0}\right) d\left(d_{0}\right) \\
& \vec{F}_{d}^{r}\left(r^{*}, x^{*}\right)=\frac{3}{2} I_{2} \int_{0}^{\infty}\left|J\left(d_{0}\right)\right|^{-1} N\left(d_{0}\right) d\left(d_{0}\right)
\end{aligned}
$$

where

$$
I_{1}=\frac{27}{8} \pi \int_{0}^{1}\left(V_{x}\left(\tau^{*}\right)-U_{x}\left(r\left(\tau^{*}\right)\right)\right) d \tau^{*} \quad I_{2}=\frac{27}{8} \pi \int_{0}^{1} V_{r}\left(\tau^{*}\right) d \tau^{*}
$$


Involving again the relation (33) we can restore the dimensional quantities

$$
\begin{aligned}
& \tilde{\vec{F}}_{d}^{x}\left(r^{*}, x^{*}\right)=\frac{9}{\pi} \tilde{\rho}_{g} \tilde{v}\left|V_{x}^{0}\right| \frac{\tilde{V}_{s}}{\tilde{k}_{e v}} I_{1} \int_{0}^{\infty}\left|J\left(\tilde{d}_{0}\right)\right|^{-1} \tilde{d}_{0} \tilde{f}\left(\tilde{d}_{0}\right) d\left(\tilde{d}_{0}\right) \\
& \tilde{\vec{F}}_{d}^{r}\left(r^{*}, x^{*}\right)=\frac{9}{\pi} \tilde{\rho}_{g} \tilde{v}\left|V_{r}^{0}\right| \frac{\tilde{V}_{s}}{\tilde{k}_{e v}} I_{2} \int_{0}^{\infty}\left|J\left(\tilde{d}_{0}\right)\right|^{-1} \tilde{d}_{0} \tilde{f}\left(\tilde{d}_{0}\right) d\left(\tilde{d}_{0}\right)
\end{aligned}
$$

The above discussed integral properties of the spray are very important practically. For example, it is shown in [9] that the rate of heat extraction from the smoke layer by the spray (directly proportional to the spray vaporization rate) determines the possibility of flashover control in compartment fires.

The studies [10,11] deal, on the other hand, with the practically important problem of smoke layer destabilization by water sprinkler or water mist systems. Possibility of such destabilization is determined by the magnitude and spatial distribution of the drag force exerted on the smoke layer by the spray.

In both cases, the presented theory offers more accurate predictions compared to simple spray dynamics models [9-11].

The importance of possessing direct formulas for integral spray properties must also be emphasized in the perspective of computing similar properties using CFD models. Accurate calculation of local spray properties (such as vaporization rates and drag forces) in CFD models is cumbersome as it typically involves counting droplet trajectories crossing particular control volumes. The number of trajectories required for statistically accurate calculation of local properties (especially at numerous locations) easily becomes prohibitive. What is worse, the investigator does not know a'priori what total number of trajectories $\mathrm{s}(\mathrm{he})$ needs to generate in order to obtain sensible number of trajectories at a given location.

All this difficulties are circumvented with the analytical formulas for integral properties which would allow easy calculation with any desirable accuracy.

\section{CONCLUSIONS}

The present paper describes a step towards development of an analytical model of water spray dynamics in fire flows that would have essential features of the field model.

In particular, in the present model we allow for the background fluid flow variation in two spatial dimensions. Further, evaporation process of droplets is taken into account and fully coupled with the droplet dynamics. Drag coefficient, in contrast to previous analytical models, varies with the Reynolds number in the full range of practically important values of the latter.

Analytical solutions for the lagrangian equations governing spray dynamics are developed and discussed. Solutions are validated against trajectories obtained by direct numerical integration of equations of droplet motion. Comparison demonstrated good agreement in the range of parameters considered. Accuracy can be further improved by applying piecewise approximation for the drag coefficient dependence on Reynolds number.

As one of applications of the proposed model, estimation of optimum droplet size for fire-fighting water sprays is considered.

Finally, explicit formulas for the integral properties of water spray, namely the density of local vaporization rate, and the local drag force are obtained.

The model will be subjected to progressive development with the view of further relaxing underlying assumptions. 


\section{REFERENCES}

[1] Novozhilov, V., (2001) Computational Fluid Dynamics Modeling of Compartment Fires, Progress in Energy and Combustion Science 27(6): 611-666.

[2] Kim, S.C., and Ryou, H.S., (2004) The Effect of Water Mist on Burning Rates of Pool Fire, Journal of Fire Sciences 22: 305-323.

[3] O'Grady, N., and Novozhilov, V., (2009) Large Eddy Simulation of Sprinkler Interaction with a Fire Ceiling Jet, Combustion Science and Technology 181: 984-1006.

[4] Nmira, F., Consalvi, J.L., Kaiss, A., Fernandez-Pello, A.C., and Porterie, B, (2009) A Numerical Study of Water Mist Mitigation of Tunnel Fires, Fire Safety Journal 44:198-211.

[5] Consalvi, J.L., Porterie, B, and Loraud, J.C., (2002) A Formal Averaging Procedure for Radiation Heat Transfer in Particulate Media, International Journal of Heat and Mass Transfer 45: 2755-2768.

[6] Collin, A., Boulet, P., Parent, G., Vetrano, M.R., and Buchlin, J.M., (2008) Dynamics and Thermal Behaviour of Water Sprays, International Journal of Thermal Sciences 47: 399-407.

[7] Meredith, K., Xin, Y., and De Vries, J., "A Numerical Model for Simulation of Thin-Film Water Transport over Solid Fuel Surfaces", Fire Safety Science -- Proceedings of the Tenth International Symposium, International Association for Fire Safety Science, 2011, pp. 415-428.

[8] Xia, J., Luo, K.H., and Kumar, S., (2008) Large-Eddy Simulation of Interactions Between a Reacting Jet and Evaporating Droplets, Flow, Turbulence and Combustion 80:133-153.

[9] Novozhilov, V., (2001) Flashover Control Under Fire Suppression Conditions, Fire Safety Journal 36: 641-660.

[10] Zhang, C.F., Chow, W.K., Huo, R., Zhong, W., and Li, Y.Z., (2010) Experimental Studies on Stability of Smoke Layer with a Sprinkler Water Spray, Experimental Heat Transfer 23(3): 196-216.

[11] Li., K.Y., Sun, X.Q., Huo, R., Wang, P., “A New Criterion for Stability of Smoke Layer under Sprinkler Spray", Proceedings of the Seventh Asia-Oceania Symposium on Fire Science and technology, Asia-Oceania Association for Fire Science and Technology, 2008, pp. 717-726.

[12] Kuo, K.K., Principles of Combustion, John Wiley and Sons, New York, 1986, p. 582.

[13] Faeth, G.M., (1983) Evaporation and Combustion of Sprays, Progress in Energy and Combustion Science 9: 1-76.

[14] Grant, G., Brenton, J., and Drysdale, D., (2000) Fire Suppression by Water Sprays, Progress in Energy and Combustion Science 26: 79-130.

[15] Ohkubo, H., and Nishio, S., (1990) Study on Accurate Prediction of Heat Transfer Characteristics of Mist Cooling, International Journal of Japanese Society of Mechanical Engineers, Series II 33 (2): 326-332.

[16] Kaleta, A., (1986) Effect of Drop Size on Extinguishing Effectiveness of a Water Spray, Archiwum Combustionis, 6(3-4): 201-212.

[17] Karlsson, B., and Quintiere, J.G., Enclosure Fire Dynamics, CRC Press, Boca Raton, 2003. 